\title{
Abernethy Malformation - Congenital Extra-hepatic Portosystemic Shunt Associated with Multiple Liver Adenomatosis: Case Report
}

\author{
Guilherme Jose de Paula Oliveira ${ }^{*}$, Samuel Ferreira, Andre Barbosa \\ Felicio Rocho Hospital, Brazil
}

Copyright $@ 2019$ by authors, all rights reserved. Authors agree that this article remains permanently open access under the terms of the Creative Commons Attribution License 4.0 International License

\begin{abstract}
In this article we report a case of Abernethy malformation, also known as congenital extrahepatic portosystemic shunt (CEPS). It is a rare vascular malformation in which the portal vein drains into a systemic vein, diverting it from its normal path to the liver. The clinical presentation is variable and most commonly the shunt is diagnosed during the propaedeutic for investigation of the symptoms. Discussion and diagnosis: R.B.S.R, 21 years old, male, with history of liver disease and previous diagnosis of multiple liver adenomatosis from 9 years of age, was hospitalized for pre-transplant liver evaluation. In the medical history, he presented delayed neuropsychomotor development, congenital scoliosis, neurogenic bladder and single kidney, and had also undergone two surgeries. His initial laboratory tests showed increased transaminases and canalicular enzymes. Ultrasonography, radiography, computed tomography (CT) and magnetic resonance imaging (MRI) were performed, which enabled us to identify and confirm important points for diagnosis. Conclusions: It is important that the radiologist recognize the findings early. CT and MRI are fundamental in the management of the syndrome, since they provide the information for diagnosis, planning, intervention and follow-up, as well as the identification of complications.
\end{abstract}

Keywords Abernethy Malformation, Hepatic Adenomatosis, Radiology, Computed Tomography, Magnetic Resonance, Ultrasound

\section{Introduction}

Described first by the surgeon John Abernethy in 1793, after performing an autopsy (Figure 1) on a 10-month-old girl that revealed termination of the portal vein (PV) in the inferior vena cava (IVC) at the level of the renal veins (1), the congenital absence of portal vein, (with presence of intra-hepatic or extrahepatic portosystemic shunt) is an extremely rare entity.

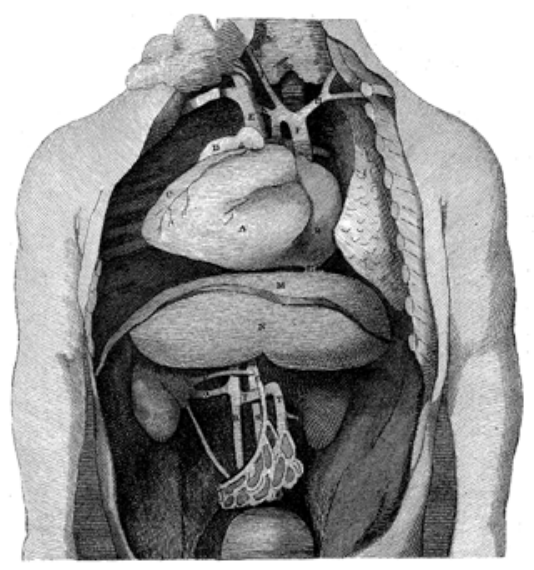

Figure 1. John Abernethy original work in 1797

Despite the increase in the number of cases diagnosed, until the moment of publication of this report, just over 300 cases were described throughout the medical literature since its discovery. The Abernethy malformation, also known as congenital extra-hepatic portosystemic shunt (CEPS), is a rare vascular malformation in which the portal vein drains into a systemic vein, diverting it from its normal path to the liver. Congenital portosystemic venous shunts are best classified into intrahepatic and extrahepatic varieties. In the first one, the connections are created between branches of the Portal vein, after its division, and the hepatic veins or Inferior Vena Cava. In extra-hepatic shunts, the anastomoses are established between the portomesenteric vasculature, before division of the $\mathrm{PV}$, and a systemic vein. Intrahepatic shunts can undergo spontaneous closure during the first year of life but the percentage of the cases in which this occurs is unknown. In the other hand extra-hepatic shunts almost always require 
intervention.

The Abernethy malformation clinical presentation is variable and most commonly the shunt is diagnosed in the propaedeutic for investigation of the symptoms. It is frequently associated with nodular liver lesions and congenital heart defects; moreover, it may cause liver dysfunction and hepatic encephalopathy. Two CEPS subvariants have been described according to the presence or absence of intrahepatic portal vein supply and the differentiation between them is essential in determination of the definitive method of treatment.

In this report, we present a case of Abernethy malformation associated with hepatic adenomatosis and a constellation of findings that has been often described as associated with this syndrome.

\section{Case Report}

R.B.S.R, male, 21 years old, natural from Juatuba - state of Minas Gerais, Brazil, with a history of hepatic disease and with previous diagnosis of multiple liver adenomatosis (confirmed by biopsy) since 9 years old, was admitted to our radiology department for hepatic pre-transplant evaluation. He had been complained of pain in right hypochondrium for more than two years as well as pruritus. In his previous medical history, he presented neuropsychomotor development delay, congenital scoliosis, neurogenic bladder, and single kidney. He had been submitted to two surgical interventions, first due to a bowel volvulus in 2012, and then a cystectomy in 2013.Besides that, he presented hepatic nodule hemorrhage in July of 201. Family history was negative for neoplasia or liver disease.

His initial laboratory tests were unremarkable except for increased transaminases and canalicular enzymes: Erythrocytes: 2, 94; Hb: 9, 4; Ht: 27,7; Glucose: 9400; Platelets: 383000; AST: 132; ALT: 56; GGT: 353; Alkaline phosphatase: 397; Cr: 0,36; Urea: 14.

Initial ultrasonographic evaluation showed the presence of multiple hepatic circumscribed isoechogenic nodules and splenomegaly. Additionally, the portal vein was absent during Doppler evaluation (Figure 2).

Radiography confirmed important skeletal changes such as scoliosis, vertebral fusion and segmentation defects, and hip dysplasia.

Computed angiotomography (CT) evaluation characterized multiple isodense, well circumscribed and homogenous enhancement hepatic nodules, with a pattern suggestive of adenoma and regeneration nodules. The biliary tree was dilated, distorted and filled with hypodense material. The presence of extrahepatic portosystemic shunt was also observed, characterized by a trunk formed from the union of the mesenteric superior vein and the splenic vein; this trunk bypassed the liver and drained straight to inferior cava vein. Those findings were later also confirmed by Magnetic Resonance (MRI) that also evoked the possibility cholangitis associated (Figures 3-5).

The patient's clinical and imaging findings suggested a syndromic picture that could correlate with all the changes found. The possibility of Abernethy's malformation was flared.

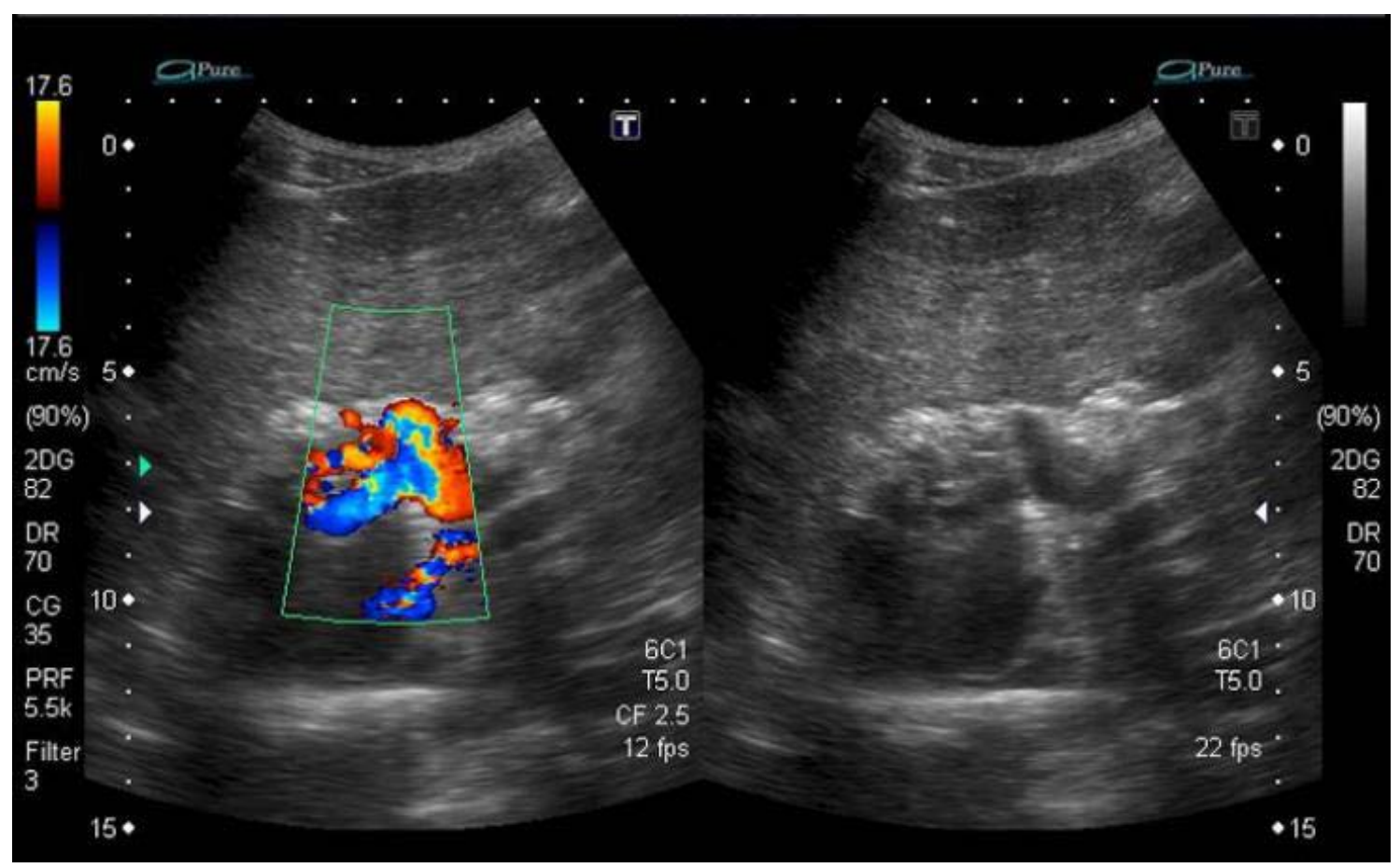




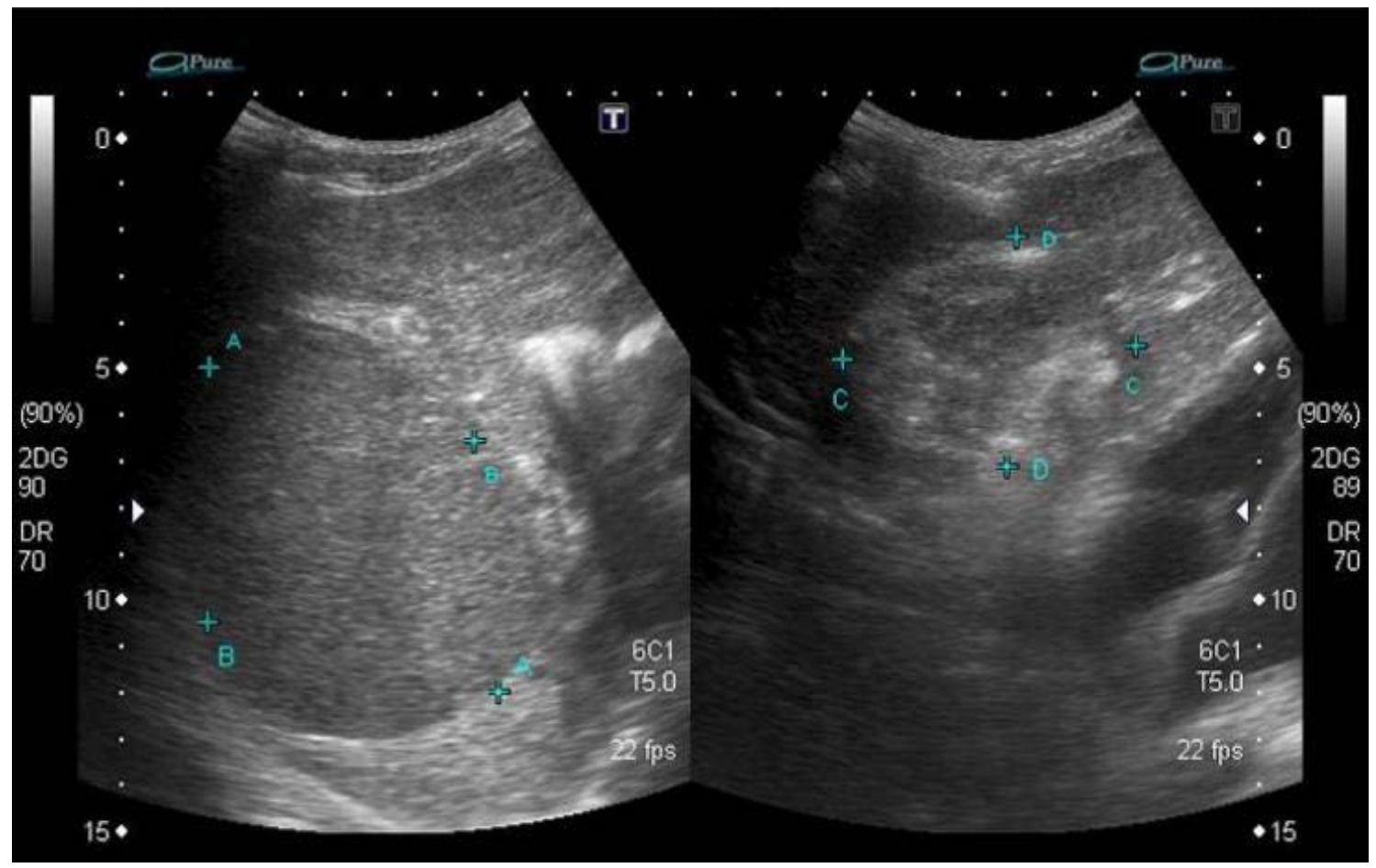

Figure 2a - 2b. The initial ultrasound revealed the presence of multiple hepatic nodules. Portal vein uncharacterized.

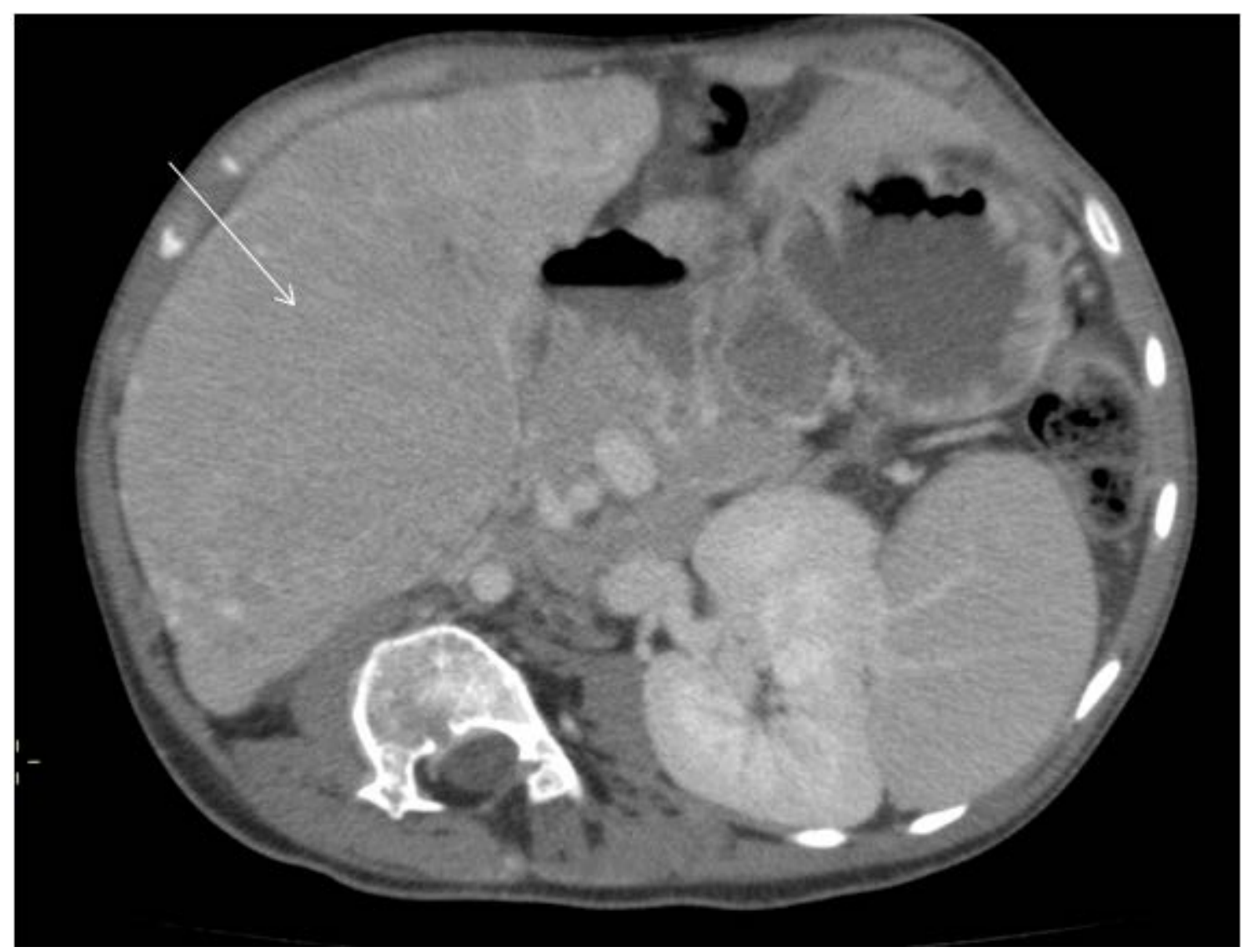



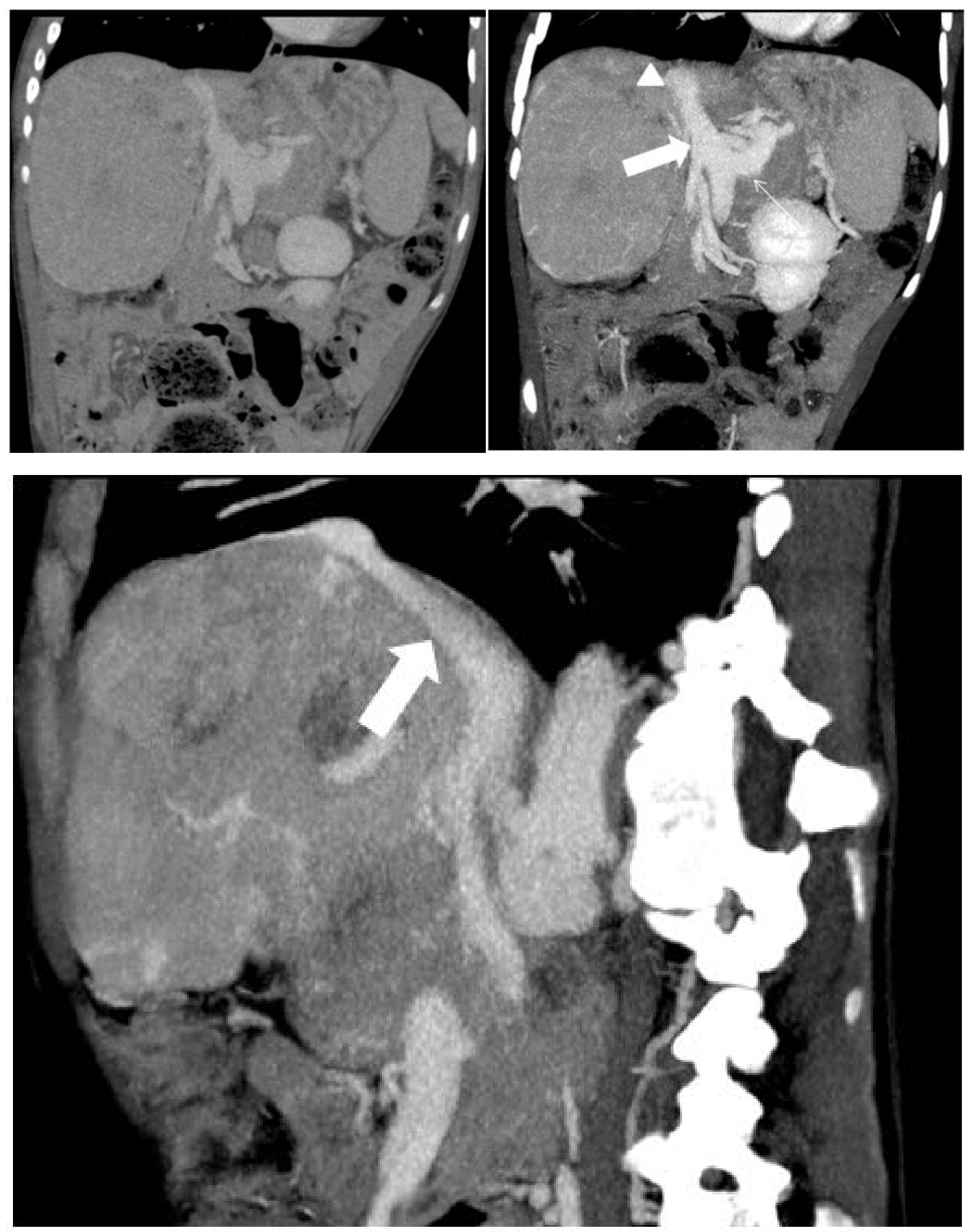

Figure 3. 3.a Contrast CT depicted multiple hepatic nodules with characteristics suggestive of adenomas; 3.b to 3.d It was also noted portal vein absence; in its place was observed a short trunk (tick arrow) formed by the union of the superior and splenic mesenteric veins (slim arrows) that "bypassed" the liver and drained directly into the inferior vena cava (arrowhead) 


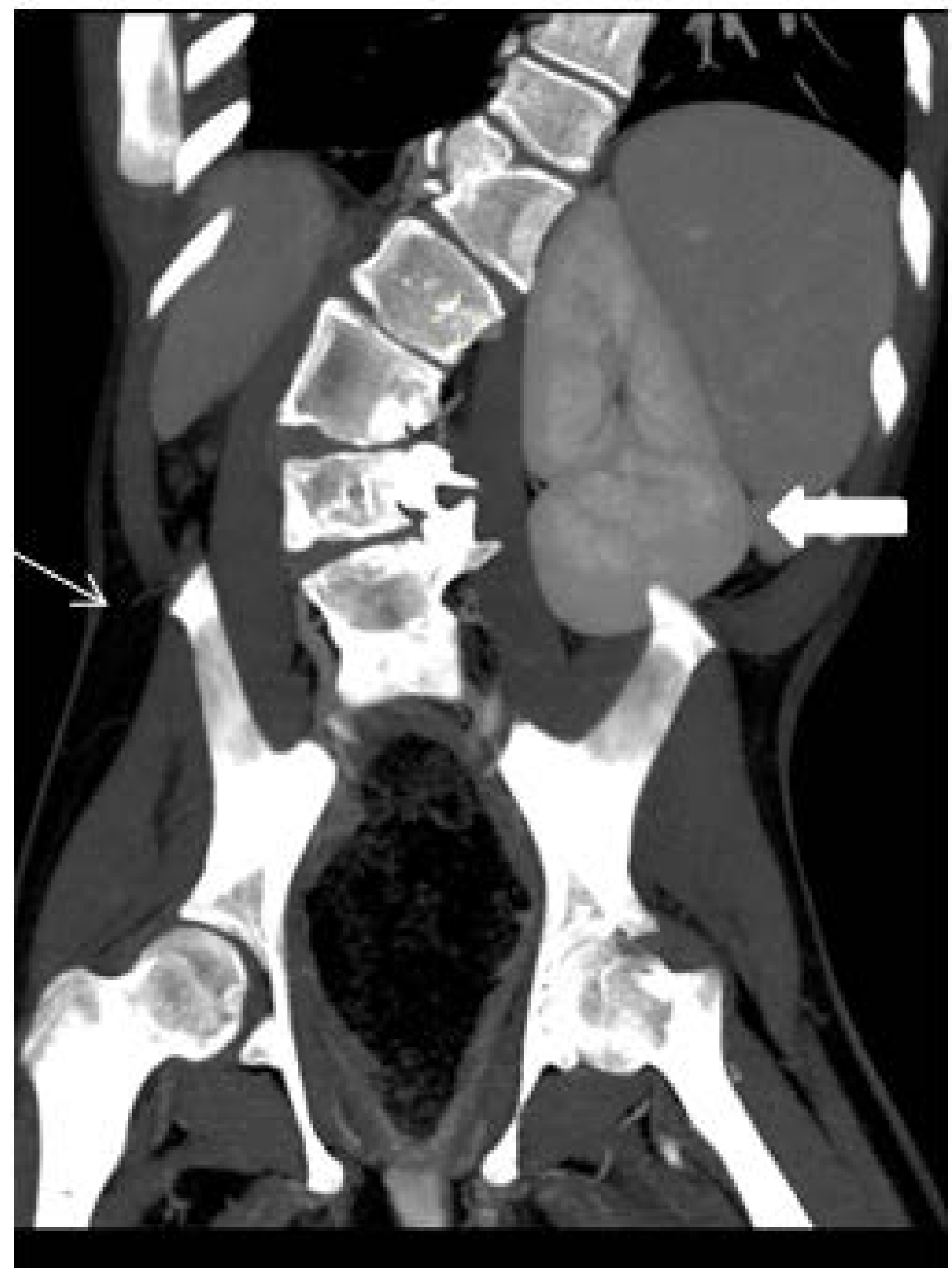

Figure 4a - 4c. Other findings included: bile tree distortion, single left t kidney (tick marrow), polysplenia, skeletal abnormalities, including segmentation and rotation defects of the vertebrae, scoliosis, and hip dysplasia (slin arrows) 


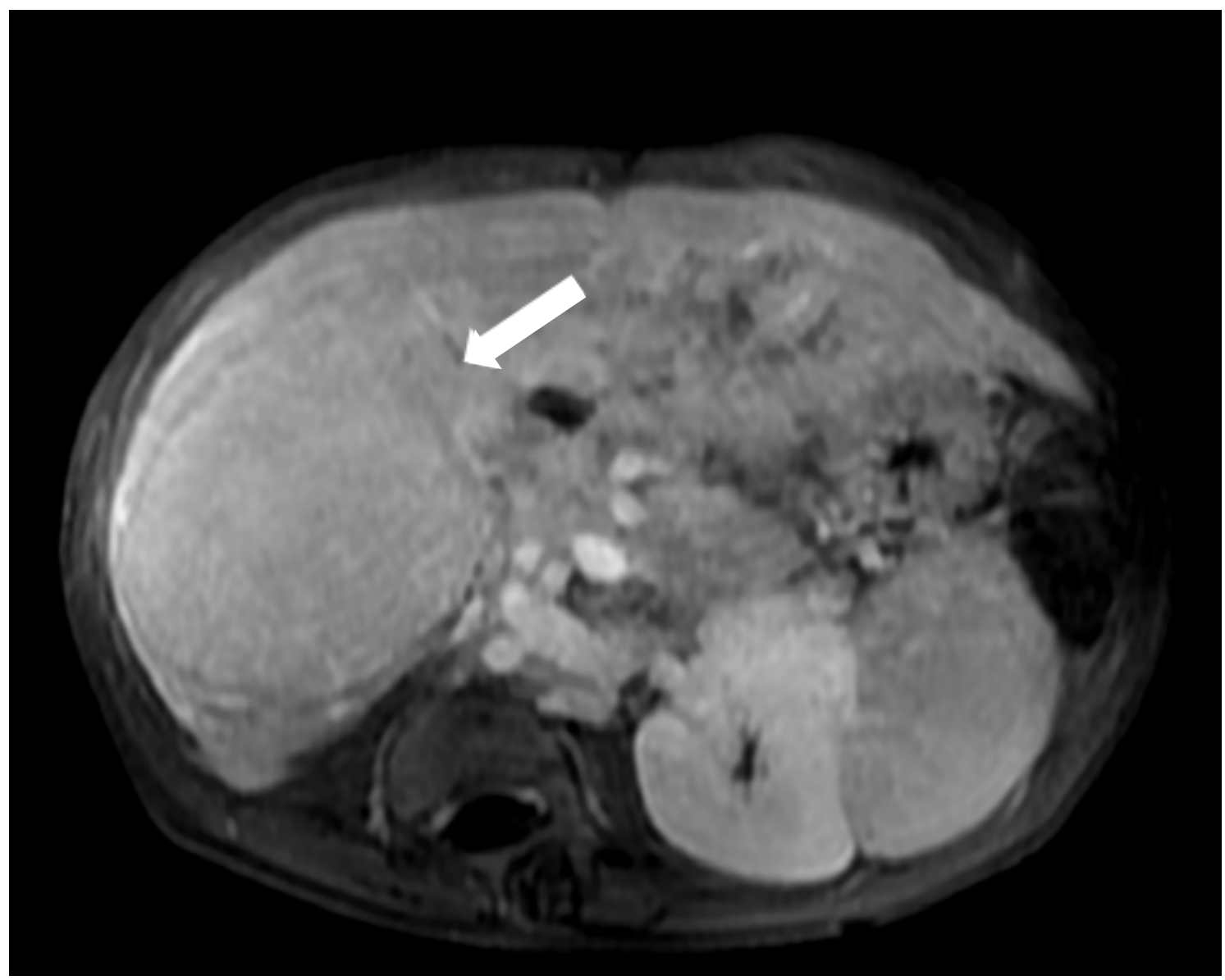

Figure 5. The findings, such as hepatic nodules (arrow), were also confirmed by MRI.

Table 1. Extra-hepatic Shunt Morgan and Superina Classification

\begin{tabular}{|l|l|}
\hline Type IA & Splenic vein (SV)m and Superior Mesenteric veins (SMV) drain separately into systemic circulation. \\
\hline Type IB & $\begin{array}{l}\text { Splenic and Superior Mesenteric veins drain together in the systemic circulation, after they converge and form a } \\
\text { common trunk. }\end{array}$ \\
\hline Type II & $\begin{array}{l}\text { Intrahepatic Portal vein (PV) is normal and the anastomosis is done through a collateral branch. } \\
\text { (Can yet be divided in 4 subtypes). }\end{array}$ \\
\hline
\end{tabular}
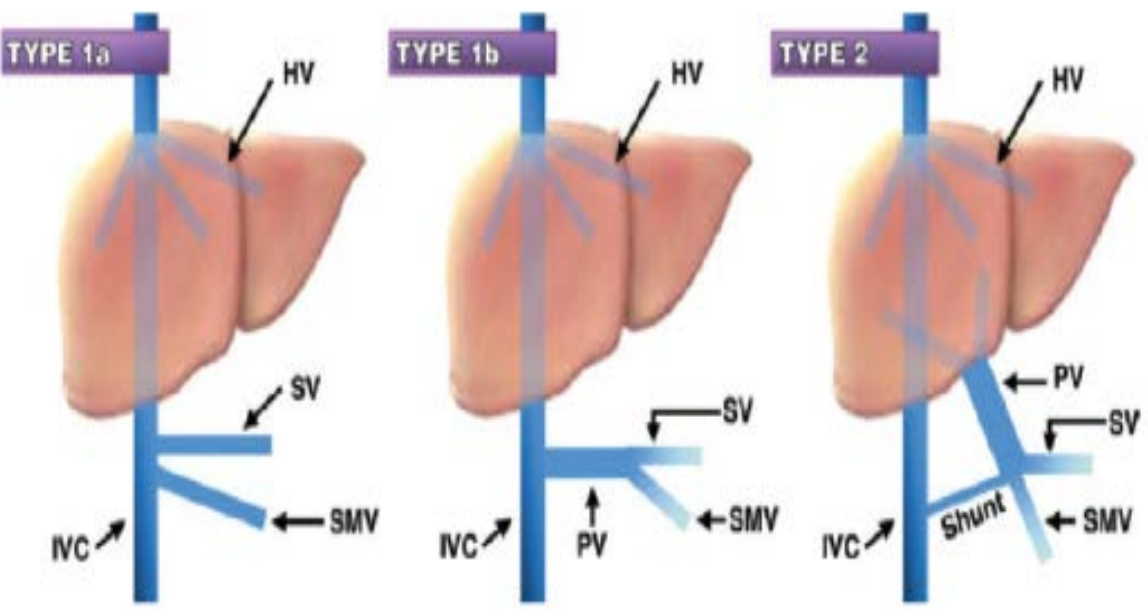

Figure 6. Extra-hepatic shunt types and associated hallmarks (Alonso and Gamarra, 2011) 


\section{Discussion}

The term Abernethy malformation has been an accepted eponym for extrahepatic portosystemic shunt (CEPS). In 1994 Morgan and Superina (3) proposed the classification that still is used for extrahepatic portosystemic shunts nowadays. This is based on the fact whether the liver is or is not perfused by portal blood and can be divided into types 2 and 1 respectively. (Table 1, Figure 6). Type 1 shunt is always congenital.

Depending on whether a trunk formed from the superior mesenteric vein and splenic vein union is present or not, two subtypes are distinguished (type 1a: missing union, type 1b: portal vein anatomical present, but with hepatic bypass). Abernethy malformation type 1 is often referred in the literature as "absent portal vein" (4). Type 2 shunt has no predilection for gender while Type 1 has a predilection for girls. Type 2 is a shunt in which the portal vein is formed, but, in addition, there is an anastomosis between the portal vein and the inferior vena cava through a patent vein remaining from the ductus venosus. They were first described by Raskin in 1964 (2), and were subclassified by Park et al, that subdivided them as follows: Type 2a: a single large vessel connecting the right portal vein to the inferior vena cava; Type 2b: a localized peripheral shunt in which one hepatic segment has one or more communications between peripheral branches of the portal vein and the hepatic veins; Type 3c: an aneurysmal communication between the peripheral portal vein and the hepatic veins; Type 4d: multiple communications between the portal vein and the hepatic veins, distributed in both lobes. (7)

Based on the classification system discussed above, we can characterize the case reported in the present study as Morgan \& Superina type 1B.

Developmental of the portal venous system is extremely complex (5). The veins of the portal system originate from the extra-embryonic and umbilical veins. The vitelline veins form a pair presenting 3 cross communication between them around the 4th week. The involution of the vitelline veins and its communicating anastomosis end up leads to portal vein formation between the 4th to 10th weeks. The pathogenesis of type 1 CEPSs has been attributed to excessive involution of the periduodenal vitelline venous loop or to total failure of the vitelline veins to establish the critical anastomoses with the hepatic sinusoids or umbilical veins (6). The persistence of the right vitelline vein originates shunts draining into the retrohepatic IVC while the persistence of left vitelline vein leads to shunts draining into the IVC or right atrium above the level of the hepatic vein confluence.

Clinically, the Alberneth's malformation is marked by a wide spectrum of symptoms that can be divided in 3 great groups: abnormal liver development, portosystemic shunt and secondary to concomitant malformations. (Table2)

Abnormal hepatic development is caused by lack of nutrition that leads to fatty degeneration and atrophy.
Usually, signs of portal hypertension are not observed.

Table 2. Clinical presentation according to CEPS types

\begin{tabular}{|l|l|}
\hline \multicolumn{1}{|c|}{ Type 1 } & \multicolumn{1}{c|}{ Type 2 } \\
\hline End-to-end anastomosis & Side-to-size anastomosis \\
\hline Congenital absence of PV & PV supple is partially conserved \\
\hline Preference for girls & No gender preference \\
\hline $\begin{array}{l}\text { Multiple associated } \\
\text { malformations }\end{array}$ & Fewer associations malformations \\
\hline
\end{tabular}

Liver growth and regeneration depends on various hepatotrophic factors such as insulin, glucagon, and epidermal growth factor, and hepatocytes deprived of portal venous blood become smaller and have reduced glycogen content. Liver of rats with congenital shunts include atrophy and hyperplastic nodules. (5) Morphological changes in hepatocytes includes decrease in their size and abundant rough endoplasmic reticulum as well an increase in cytoplasmic mitochondrial density. This can explain the development of tumors.

Around 50\% of patients have hepatic focal lesions, more commonly regeneration nodules, focal nodular hyperplasia and adenoma. Hepatomegaly can be observed due to regenerative or hyperplastic nodular changes. Biopsy is not needed most of the time; however, in patients suspected of having type 1 CEPS, a liver biopsy is needed as it may reveal small portal venules within the portal triads, a finding indicative of a type 2 shunt. Consequently, biopsy avoids that some type 2 shunts could be misdiagnosed as type 1 shunts if the diagnosis were based on imaging alone.

Malignant transformation of regenerative nodules is unusual but has been reported in association with CEPS (8). Those include hepatoblastoma and hepatocellular carcinoma. Consequently, long-term follow-up is necessary.

Portosystemic shunt causes increase in ammonia, galactose and other potentially harmful metabolites levels. Encephalopathy can occur, especially in elderly patients and it can be a big challenge. Encephalopathy develops spontaneously due to an increased sensitivity of their central nervous system to hyperammonemia or due to precipitating events such as infections. Psychiatric disorders and neuropsychological developmental delay have been also reported.

Hepatopulmonary syndrome is also other possible development (6). This syndrome is related to diversion of vasoactive mediators into the systemic circulation, leading to dilatation of intrapulmonary vessels resulting in hypoxemia due to ventilation - perfusion mismatch. Digital clubbing can be observed in those cases.

Among the congenital anomalies associated with type 1 portosystemic shunt we emphasize cardiovascular, gastrointestinal, genitourinary and skeletal, described in table 3.

Ultrasonography is the initial diagnostical test because it is non-invasive, does not expose patients to ionizing radiation harm, is cheap and available anywhere. It 
demonstrates the absence of the portal vein. Reduction of liver volume and periportal hyperechogenicity are other common findings. Doppler mode is useful in demonstrate the blood flux direction in the vessels. However, ultrasound usually fails to demonstrate the extra-hepatic shunt once they can be very small. When the CEPS diagnosis is suspected, other imaging modalities have to be ordered.

Table 3. Malformations associated with congenital portosystemic shunt.

\begin{tabular}{|l|}
\hline Cardiovascular anomalies \\
Atrial septal defects \\
Patent foramen ovale \\
Ventricular septal defect \\
Patent ductus arteriosus \\
Tetralogy de Fallot \\
Dextrocardia \\
Mesocardia \\
Congenital stenosis of aortic valve \\
\hline Gastrointestinal anomalies \\
Polysplenia \\
Biliary atresia \\
Choledochal cyst \\
Intrahepatic gallbladder \\
\hline Genitourinary anomalies \\
Multicystic dysplastic kidney \\
Bilateral ureteropelvic stenosis \\
Vesicoureteral reflux \\
Crossed fused renal ectopia \\
Hypospadia \\
\hline Skeletal anomalies \\
\hline Cutaneous vascular malformation and tumors \\
\hline Vascular anomalies \\
Interruption of IVC \\
Double IVC \\
\hline
\end{tabular}

Both CT angiography and MR angiography allow identification of the absent portal branches. CT is superior in the detection of anastomotic small branches. MRI is considered the method of choice. Regenerative nodules are usually multiple and have a well-defined appearance usually ranging in diameter from 0.5 to $4 \mathrm{~cm}$. At MR imaging, they typically have high signal intensity on T1-weighted images (75\% of cases). It is isointense or slightly hyperintense on T2-weighted images. (6).

Because of the absence or reduction of portal blood, the hepatic arterial phase is dominant in contrast-enhanced CT or MR imaging. Usually, regenerative nodules demonstrate homogeneous enhancement and unlike some other focal hepatic lesions, usually remain hypervascular with slight hyperintensity in the portal venous dominant phase of bolus-enhanced MR imaging.

Conventional angiography is the gold standard for the study of portomesenteric vasculature, but it has the disadvantage for been invasive, for the radiation exposure and for require anesthesia. Indirect mesenteric portovenography is the angiographic technique used. This technique is performed during the visceral phase of mesenteric arteriography. When it fails to provide a distinct image of the shunt, percutaneous transhepatic portography may be required. Advantages of the conventional angiography include selective embolization of shunts, what is a possible therapy for type 2; transvenous liver biopsy, and measurement of the pressure gradients.

Nuclear medicine studies are useful for calculating the shunt ratio in type 2 CEPS, an important factor in determining the age at which the onset of encephalopathy occurs. The shunt ratio can be determined with portal scintigraphy performed with rectal administration of iodine (123I) iodoamphetamine. The isotope is absorbed into the inferior mesenteric vein and carried to the liver. In patients without liver shunts, only liver images are captured; however, if a portosystemic shunt is present, the isotope is detected in the liver and lungs simultaneously. So, the ratio between lung and liver capitation can be calculated. Usually, patients with shunt ratios of 30\%-60\% are more probable to develop encephalopathy after precipitating events, whereas those with shunt ratios of greater than $60 \%$ (especially older patients) are at high risk for developing spontaneous encephalopathy. This information can be used to plan when to take further intervention.

For definitive diagnosis of CEPS, potential acquired portosystemic shunts must be excluded and this includes patients with imaging findings of cirrhosis, with or without concomitant features of portal hypertension, and surgically created portosystemic shunts (6). An important feature is CEPS generally do not present with signals of portal hypertension. This is important in distinguish the absence of portal vein observed in CEPS from the absence that can be found in chronic thrombosis. Other differences include vessel enlargement, calcifications e cavernomatous transformation in chronic portal thrombosis. However, this differentiation is not always easy.

Spontaneous closure of a CEPS has not been reported until now. Treatment varies according to shunt type and clinical symptoms. Children are more resistant to hepatic encephalopathy than are adults, and most are asymptomatic. Pharmacotherapy and dietary measures such as a reduced-protein diet are the initial approach. Determination of the type of shunt is particularly important for the planning of definitive treatment. In patients with type 1 CEPS, occlusion of the shunt is not an option as it represents the only drainage route for the mesenteric and splenic venous blood; liver transplantation is treatment of choice. CEPS type 2, shunt occlusion (surgical or through embolization) is among the treatment options. Regeneration after resection of tumors generally is completely satisfactory, and this anomaly does not appear to represent a contraindication to liver surgery.

\section{Conclusions}

Malformation of Abernethy is a diagnostic challenge given its rarity as well as the range of associated results. The clinical presentation is variable and more commonly is diagnosed during the propaedeutic for the investigation of the symptoms. Other congenital anomalies can be found in 
patients, like, for example, congenital heart problems and skeleton anomalies; hepatic nodules are common and can be single or multiple. The CEPS type was defined according to the presence or absence of intrahepatic portal vein and the differentiation between the two variants, is determinant for the correct treatment. In our case report we had the opportunity to analyze a Morgan and Superina type $1 \mathrm{~B}$ variant. Computed tomography and magnetic resonance imaging are fundamental in the management of the syndrome, since it is required information for diagnosis, planning, intervention and follow-up, as well as identification of complications. It is crucial that radiologists recognize the findings early.

\section{Acknowledgements}

We disclose no conflict of interests in this article.

\section{REFERENCES}

[1] Abernethy J (1793) Account of two instances of uncommon formation in the viscera of the human body. Philos Trans $\mathrm{R}$ Soc Lond B Biol Sci 83: 59-66

[2] Tiziana Timpanaro, Stefano Passanisi, Alessandra Sauna, et al., "Congenital Portosystemic Shunt: Our Experience," Case Reports in Pediatrics, vol. 2015, Article ID 691618, 5 pages, 2015. doi:10.1155/2015/691618

[3] Morgan G, Superina R. Congenital absence of the portal vein: two cases and a proposed classification system forportasystemic vascular anomalies. J Pediatr Surg 1994; 29(9):1239-1241.

[4] Ringe K, Schirg E, Melter M et-al. [Congenital absence of the portal vein (CAPV). Two cases of Abernethy malformation type 1 and review of the literature]. Radiologe. 2008; 48 (5): 493-502.

[5] Howard ER, Davenport M. Congenital extrahepatic portocaval shunts: the Abernethy malformation. J Pediatr Surg 1997; 32(3):494-497.

[6] Alonso-Gamarra E, Parron M, Perez A, Prieto C, Hierro L, Lopez-Santamatia M (2011), Clinical and radiological manifestations of congenital extrahepatic portosystemic shunts: a comprehensive review.

[7] J. H. Park, S. H. Cha, J. K. Han, and M. C. Han, "Intrahepatic portosystemic venous shunt," American Journal of Roentgenology, vol. 155, no. 3, pp. 527-528, 1990.

[8] Murray CP, Yoo SJ, Babyn PS. Congenital extrahepatic portosystemic shunts. Pediatr Radiol 2003; 33 (9):614-620. 